\title{
Children's Choice of Games: The Influence of Prosocial Tendency and Education-Level
}

\author{
Vivian Hseuh-Hua Chen ${ }^{1}$, Weirong Lin $^{1}$, Chiew Woon $\mathrm{Ng}^{1}$, Su Li Chai ${ }^{2}$, \\ Angeline Cheok Eng Khoo ${ }^{2}$, and Henry Been-Lirn Duh ${ }^{3}$ \\ ${ }^{1}$ Wee Kim Wee School of Communication and Information, 31 Nanyang Link, \\ Nanyang Technological University, Singapore 637718 \\ \{chenhh, wrlin, chiewwoon\} @ntu.edu.sg \\ ${ }^{2}$ Psychological Studies Academic Group, National Institute of Education, \\ 1 Nanyang Walk, Singapore 637616 \\ \{suli.chai, angeline.khoo\} @nie.edu.sg \\ ${ }^{3}$ Department of Electrical and Computer Engineering, National University of Singapore, 4 \\ Engineering Drive 3, Singapore 117576 \\ \{eledbl\}@nus.edu.sg
}

\begin{abstract}
This study employed the uses and gratifications approach to examine children's choice of gaming genres. The measure of prosocial behavioral tendency was used as an approximation of a child's offline gratification, and this was related to the exposure to three different genres of games (violent, aggressive and prosocial). The influence of education level was also taken into consideration. Data was compiled and analyzed from a survey conducted on Singaporean schoolchildren $(N=2,640)$. Overall results supported the supplementary model of gratification seeking behavior. Children with higher prosocial scores spent significantly less time playing violent and aggressive games, whereas children of a higher education level spent more time playing games of all genres. The results are presented and discussed.
\end{abstract}

Keywords: Uses and Gratifications, Games, Prosocial Orientations.

\section{Introduction}

With the fast increasing popularity of games, scholars are now beginning to take note of gaming as a media phenomenon deserving of their attention. Though more focus has been paid to the weightier issues such as game addiction and the negative effects of games, there is now a widening of the scholarly scope to include other issues such as motivations for gameplay and the social, economic and cultural aspects of virtual communities.

Besides improving the marketability of games, research into gameplay motivations and choice of games also enables a greater understanding of the appeal of games and how gameplay can be seen as an extension or a supplement to the real-life activities of gamers. Children are especially perceived to be easy prey for game developers, who market addictive virtual play-worlds that are targeted at specific age ranges. However, though it would be simple to portray children as largely passive consumers of games, 
the reality might not be so. There is a need to approach the consumption of games by children through a perspective that treats them as active consumers of media, which this study seeks to do via the uses and gratifications approach. To begin, the following literature review first traces the development of media theory, documenting the shift from the notion of a passive audience to that of an active one.

\section{Literature Review}

\subsection{Media Effects Models and the Uses and Gratifications Approach}

Early models of media effects emphasized the passivity of audiences, with the development of theories such as the hypodermic syringe or magic bullet theory. These perspectives viewed media consumption as an activity whereby the media exerted a unilateral influence on its viewers. Hence, its effects were conceived as something similar to how a syringe injects ideas directly into the consciousness of the masses.

Later on, developments in media effects theories shifted the prevailing consensus to give greater prerogative to individual agency. This newer approach could be traced back to the Lazarsfeld studies on media effects, which concluded that the media seldom had strong effects [1]. Following this, other scholars began rethinking the dominant, strong effects paradigm. Katz and Foulkes trace the development of the uses and gratifications approach from the 1940s onwards, noting how uses and gratifications research really became fruitful once researchers began to understand individual media use by turning to the social-psychological attributes of individuals [2]. These perspectives later matured into what is now known as the uses and gratifications paradigm, which represents a new way of thinking about the influence of media. Sharing somewhat similar premises with the psychological perspective of rational choice theory, the uses and gratifications approach emphasizes how individuals consume the media with the intention to obtain certain kinds of fulfillment.

However, scholars have also drawn attention to the potential drawbacks of the uses and gratification approach. For instance, some note that there is not yet a unified understanding of the approach, and many central concepts of the theory have not been made clear [3]. Others state that by focusing on audience consumption, the approach tends to be too individualistic [4]. Despite these limitations, media scholars suggest that these models still provide a useful framework from which to study communication, especially the issues surrounding the Internet and new media $[3,5,6]$.

\subsection{A Uses and Gratifications Approach to Gameplay}

Employing a uses and gratifications approach essentially involves analyzing media choice by focusing on the notion of gratifications, or needs, that media use satisfies [3]. By identifying the motivations and traits behind individuals' choice of a particular media, the effects of media can then be viewed as a function of its use [7]. The perspective has been fruitfully employed in a range of media studies, especially those focusing on television usage. Yet, studies that have explicitly applied the approach to understanding gaming behavior have been few. Sherry and colleagues traced the studies on gaming that have used the uses and gratifications approach in the past [7]. According to the authors, Selnow's study [8] on gaming that adapted a television uses 
and gratifications scale was probably one of the earliest to investigate gaming practices. Their factor analysis yielded five key points, that (1) gameplay was preferable to human companionship, (2) it taught about people, (3) it provided companionship, (4) activity, and (5) escape. Another study by Wigand et al. [9] employed a similar approach found that gameplay was desired as it provided the necessary gratifications of excitement, satisfaction of doing well, and tension-reduction. More recently, studies by three groups of researchers [10- 12] have revealed gratifications such as passing time, enjoyment, skill testing, stress reduction, and competition [7].

Scholars have also used the uses and gratifications approach to link online and offline behavior. Scholars describe what is known as a "deficit model" (Steinkuehler, as cited in [13]) which refers to how the absence of a gratification in player's real lives drives them to seek it elsewhere. For instance, Burgoon [14] found that talk radio callers expressed less interest in communicating face-to-face, perceiving it to be less rewarding. It is possible that these individuals turned to talk radio to compensate for their lack of interest in face-to-face communication. In another study, individuals who perceived interpersonal interaction to be unrewarding turned to interaction based on Internet communication, hence perceiving it as a suitable alternative [15].

On the other hand, past research (e.g., of Lee and colleagues [13]) suggests instead that there may exist a supplementary model, where players are driven to seek more intense sources of gratification for a similar kind of need. The authors found significant positive correlations between gratifications obtained and sought in players' real and online (i.e., gaming) lives, thus supporting their claim. Similarly, Bruning [16] related motives for interpersonal and computer-mediated communication, finding that people enjoyed both interpersonal as well as computer-mediated-communication. This could be interpreted as an indication of how the fulfillment of a need in offline life drives individuals to seek other avenues of similar fulfillment, such as those available through online communication.

This strand of research that seeks to link offline and online gratifications seems promising, and the present study seeks to contribute to the debate by examining how real life gratifications are related to an individual's choice of game. This study uses the psychological measure of prosocial orientation as a measurement of the gratifications sought in real life. The prosocial orientation measure has been used in previous developmental psychology studies, and has been employed in several gaming studies as well. In addition to this, this study will also examine the effect of educational level on choice of game genre.

\subsection{The Influence of Prosocial Orientation}

Various psychological theories [17, 18] have proposed that basic human needs are universal, describing them as playing a central role in human life in which the fulfillment of these needs is critical in the process of growth, development and well-being.

Basic needs can be likened to a persistent feeling of hunger. For instance, Staub [18] conceptualizes basic needs as "the most fundamental motives" (p. 52) and proposes basic human needs as the driving force behind various courses of human action. In addition to the core set of basic needs documented in psychological literature, he emphasizes needs for "effectiveness and control" - the feeling of efficiency and control of events and situations; "positive identity" - positive self-conception and 
self-esteem (through enhancement of esteem from others); "positive connection to other individuals" - ownership of relationships which are positively connected to other individuals or groups; and "transcendence of the self" - the need to go beyond the self, which can all be satisfied by helping others (p. 56). For instance, if the act of engaging in helpful behavior has positive connotations in one's culture and/or assists in the improvement of the welfare of others, not only would it be able to satisfy these needs of "positive identity," "positive connection to others" and "effectiveness and control," it would also be regularly affirmed within a community. This promotes an internalization of already developed values, beliefs and principles through social experiences, effectively leading individuals to cherish others' welfare and view themselves as helpful persons. Hence, through the pursuit of basic human needs fulfillment, prosocial orientation and values are promoted, fostering the individuals' prosocial behavioral tendencies.

It should be noted that the bulk of previous studies have viewed these prosocial behavioral tendencies as largely a consequence of, and not an antecedent to, exposure to gaming. For instance, an individual's prosocial tendency is construed to be a result of his or her exposure to certain genres of games. While that interpretation is widely held, it is equally likely that these behavioral tendencies exert some form of effect on the choice of gaming genres. This study approaches the issue by taking prosocial behavior as chronologically antecedent to choice of game genre, and employs the psychological measure for individual prosocial behavioral tendency that is derived from Cheung et al's work [19]. In particular, the helping behavior subscale will be used as an approximation of an individual's gratification seeking behavior offline.

\subsection{Education Level and the Development of Prosocial Orientation}

In considering the effect of prosocial orientation on the choice of game genre, previous literature draws some attention to the influence of education level on the development of prosocial tendencies in children. Advancements in childrens' ability to engage in moral reasoning are concomitant with the ability to see reality through the perspective of another, as well as the ability to grasp abstract concepts [20]. The development of prosocial behavior has also been documented to occur in tandem with advancement in education level and age of children. Eisenberg [20] described how elementary school children exhibit moral behavior primarily to seek approval or by adhering to stereotypical models of good. Throughout elementary school, direct reciprocity reasoning develops, where children exhibit good behavior as a means to self-gain (when others reply in kind). Finally, as late elementary school gives way to middle school and beyond, children begin to reason using morally abstract notions and they develop emotional responses to behavior (such as guilt), and acquire the ability to take on the perspective of others.

These differences in the moral development of the child affect the levels of prosocial behavioral tendency, especially in the key transition stage between late elementary school and early middle school. Development in moral reasoning may affect the degree of prosocial orientation, or at the very least alter the reasons underlying the prosocial orientation held to by the child. Hence, this study also takes into consideration the influence of level of education on the choice of game genre, through its effect on the development of the child's prosocial orientation. 


\subsection{Research Questions}

The present study examines the issue of gameplay using the uses and gratifications approach, by analyzing the links between individual prosocial behavioral tendency and the choice of game genres. This study will also examine the influence of the level of education on the choice of game. Hence, the following research questions are proposed:

RQ1: How does the degree of prosocial orientation affect the choice of game genre? RQ2: How does the level of education of the child affect the choice of game genre?

\section{Method}

\subsection{Participants}

Participants were $(N=2,640)$ students from twelve primary and secondary schools in Singapore in which four of these schools were boys-only schools: 1329 primary school children (956 males and 372 females) and 1311 secondary school children (1004 males and 302 females). The age of primary school children ranged from 8 to 12 with the median age at 9 years. The ethnic composition of this sample was: $67.7 \%$ Chinese, $13.2 \%$ Malay, $8.6 \%$ Indian, 1.4\% Eurasian, and 2.9\% other. The median age for secondary school children was 13 and the ages ranged from 12 to 16 . The ethnic composition for this sample was: $71.5 \%$ Chinese, $13.3 \%$ Malay, $6.9 \%$ Indian, $0.6 \%$ Eurasians, and $3.4 \%$ other.

\subsection{Procedures}

The questionnaires were administered to children collectively at school during March to May 2007. These questionnaires were counterbalanced in various orders. Children were measured on their prosocial behaviors and their video gaming habits.

\subsection{Measures}

An eleven-item subscale from Prosocial Orientation Questionnaire (POQ) [19] was used to measure children's helping behavior. Sample questions of helping behavior include "I would give up something to help my friends or family" and "I would volunteer to help a charity if they need my help". Children rated their agreement to these items on a scale from 1 (strongly disagree) to 4 (strongly agree). The scores were transformed to Percent of Maximum Possible (POMP) that ranged from 0 to 100. The primary school children mean sample for helping behavior was 70.86, standard deviation was 16.18 and the alpha was 0.73 ; while the secondary school children mean sample was 65.71 , standard deviation was 13.30 and the alpha was 0.73 . Children who scored above the mean were categorized as highly prosocial oriented, whilst children who scored below the mean were classified as low prosocial oriented.

Children were asked to list three of their favorite games and reported the estimated time they spent on each of these games (in hours per week). To measure the prosocial content of the game, two questions were included: "How often other players help each other in the game" and "How often they help others in the game," which were rated on 
a scale from 0 (never) to 3 (almost always). For the violent content of the game, another two questions were included: "How often do they shoot or kill creatures in the games" and "How often do they shoot or kill other players in the game" were assessed. To measure the aggressive content of the game, "How often do characters try to hurt each other's feelings in the game" was used. The prosocial, violent and aggressive content of game scores were computed through multiplying across each game genre's ratings and the amount of time spent playing the game. These scores were then averaged across all three aforementioned games [22] and were standardized.

This study chose to interpret game genre by seeking the respondents' evaluations of the activities they are involved within the game rather than allowing respondents to select from a list of pre-classified games. This gives the most accurate assessment of the specific activities that individuals are involved in as they play the game, as each game may provide a combination of several different types of gratification.

\section{Results}

The two research questions were tested through Multivariate Analysis of Variance (MANOVA), followed by a series of univariate F-tests to examine each of the dependent variables: Time Spent on prosocial $(M=1.35, S D=2.29)$, violent $(M=0.89$, $S D=2.02)$ and aggressive content games $(M=1.25, S D=2.06)$. The results are reported in separate sections for each independent variable: Children's prosocial orientation, children's education level and interaction between prosocial orientation and education level.

Table 1. Descriptives of Prosocial Orientation and Education Level on Game Genres

\begin{tabular}{lccccccccc}
\hline & \multicolumn{3}{c}{ Prosocial Orientation } & \multicolumn{3}{c}{ Education Level } \\
\cline { 2 - 10 } & \multicolumn{2}{c}{ Low } & \multicolumn{2}{c}{ High } & Primary & \multicolumn{2}{c}{ Secondary } \\
\cline { 2 - 11 } & $M$ & $S D$ & $M$ & $S D$ & $M$ & $S D$ & $M$ & $S D$ \\
\hline Violent Game & 1.64 & 2.56 & 1.06 & 1.92 & 0.97 & 2.00 & 1.75 & 1.67 \\
Aggressive Game & 1.17 & 2.35 & 0.64 & 1.42 & 0.65 & 1.78 & 1.18 & 2.14 \\
Prosocial Game & 1.39 & 2.20 & 1.09 & 2.21 & 0.89 & 1.79 & 1.61 & 2.21 \\
\hline
\end{tabular}

\subsection{Prosocial Orientation on Choice of Game Genre}

To test whether the degree of prosocial orientation affects the choice of game genre, a multivariate test was performed. The combined DVs (prosocial, violent and aggressive game content) were significantly affected by children's prosocial orientation, Wilk's Lambda, $F(3,2125)=13.35, p<0.01, \eta^{2}=0.018$. High prosocially orientated children reported spending less time playing violent content games, $F(1,2127)=$ $19.49, p<0.01, \eta^{2}=0.009$. High prosocially oriented children also reported spending 
less time playing aggressive content games, $F(1,2127)=26.16, p<0.01, \eta^{2}=0.012$. However, no significant result was found between prosocial orientation and prosocial content games.

\subsection{Education Level on Choice of Game Genre}

The MANOVA revealed that the combined DVs (prosocial, violent and aggressive game content) were significantly affected by education level, Wilk's Lambda, $F(3$, $2125)=20.80, p<0.01, \eta^{2}=0.029$. Secondary school children reported spending more time playing all three genres of games as compared to primary school children [for violent content, $F(1,2127)=46.67, p<0.01, \eta^{2}=0.021$; aggressive content, $F(1$, $2127)=24.61, p<0.01, \eta^{2}=0.011$; prosocial content, $F(1,2127)=59.53, p<0.01$, $\left.\eta^{2}=0.027\right]$.

\subsection{Interaction between Prosocial Orientation and Education Level on Choice of Game Genre}

The combined DVs were significantly affected by the interaction of children's education level and prosocial tendency, $F(3,2125)=2.95, p<0.05, \eta^{2}=0.004$. There is a marginally significant interaction between children's education level and prosocial tendency on aggressive content game, $F(1,2127)=3.00, p=0.08, \eta^{2}=0.001$. That is, though low prosocially oriented primary children $(M=0.82, S D=2.14)$ spent more time playing aggressive content games than high prosocially oriented primary school children $(M=0.53, S D=1.48)$, the discrepancy is even greater between low $(M=$ $1.40, S D=2.46)$ and high prosocially oriented $(M=0.81, S D=1.42)$ secondary school children. No other significant interaction was found.

\section{General Discussion}

Results showed that children with higher prosocial scores tend to spend significantly less time playing violent and aggressive games. Relating this finding back to the models of gratification seeking behavior described in the review of literature, this finding seems to be supportive of the supplementary model. Children seek and receive a similar kind of gratification from their activities both in their offline lives as well as in the games that they play. Correspondingly, the children with lower prosocial scores tend to spend significantly more time playing violent and aggressive games, perhaps indicating that they also enjoy behaving less prosocially in-game, just as they do in their offline lives.

Results also revealed that secondary school children spent significantly more time playing violent, aggressive and prosocial games. Although in general primary school children reported themselves to be more helpful as compared to the secondary school children (refer to Measures under Methodology), their lower levels of prosocial gaming habits seem to suggest otherwise. In the present study, it was difficult to distinguish if these prosocial content games were single-player or multiplayer online games (such as Massively Multiplayer Online Role Playing Games). If we assume prosocial games to be largely multiplayer oriented, a predominant feature of such games is 
social interaction, where players are offered many opportunities to assist others and where the game dynamics require cooperation and coordination between players to achieve certain goals. Curiously, primary school children (who were generally more helpful) did not spend as much time in these prosocial games as secondary school children. A possible explanation lies in the deficiency in gratification that these younger children obtain by participating in such games. Previous studies have shown how maturity is a vital factor to successful community integration in this genre. Players often discriminate against younger players because they are perceived to be less mature, less willing to contribute to general goals, and less cooperative [23]. This fosters an unwelcoming communal climate for younger players and makes the gaming experience significantly less socially gratifying, which explains younger players' lower exposure to games in the prosocial genre.

In addition, some of the developmental theories on prosocial behavior suggest that young children are not fully developed in their comprehension of the concept of help. According to Cialdini's Socialization Model (as cited in [24]), children between the ages of 10 to 12 (i.e., primary school children) are not fully aware of the importance or emphasis of helpfulness in the society, and only help when told to do so. The fact that primary school children in this study on average reported themselves to be quite helpful (more so than those in secondary school) might be due largely to how the importance of certain helping behaviors are frequently impressed upon them by their parents and teachers. Since the measurement of prosocial behavior is measured in simple and clear-cut scenarios, with no moral ambiguity, these children find it easy to pick out the behavior that is required of them. For instance, the prosocial questionnaire had items such as "I always think of helping people who are disabled." However, in-game helping behavior often involves complex decisions in scenarios riddled with moral grey areas. Hence, it should come as no surprise that younger children are unable to fully grasp the nature of helping behavior that unfolds in online interaction. Again, this hinders their expression of prosocial behavior and makes their gaming experience less socially gratifying, which accounts for lower rates of prosocial game exposure.

Finally, it should be noted that though this study's use of the prosocial behavioral tendency provides a novel way of approximating gratification-seeking behavior, there is a potential danger of reading too much into the behavioral tendencies of respondents. In future studies, such psychological measures should be used in combination with more traditional and straightforward methods of measuring gratification seeking behavior to obtain a comprehensive measure of the concept.

Acknowledgments. The study was funded by a grant from the Singapore Ministry of Education and Media Development Authority (EP1/06/AK).

\section{References}

1. Rogers, E.M.: A History of Communication Study. The Free Press, New York (1994)

2. Katz, E., Foulkes, D.: On the Use of Mass Media as Escape: Clarification of a Concept. Public Opinion Quarterly 26, 377-388 (1962) 
3. Ruggerio, E.T.: Uses and Gratifications Theory in the 21st Century. Mass Communication and Society 3, 3-37 (2000)

4. Elliott, P.: Uses and Gratifications Research: A Critique and a Sociological Alternative. In: Blumler, J.G., Katz, E. (eds.) The Uses of Mass Communications: Current Perspectives on Gratifications Research, pp. 249-268. Sage, Beverly Hills (1974)

5. Cho, J., Zuniga, H.G.D., Rojas, H., Shah, D.V.: Beyond Access: The Digital Divide and Internet Uses and Gratifications. IT \& Society 1, 46-72 (2003)

6. Morris, M., Ogan, C.: The Internet as Mass Medium. Journal of Communication 46, 39-50 (1996)

7. Sherry, J.L., Lucas, K., Greenberg, B.S., Lachlan, K.: Video Game Uses and Gratifications as Predictors of Use and Game Preference. In: Vorderer, P., Bryant, J. (eds.) Playing Video Games, pp. 213-224. Routledge, New York (2006)

8. Selnow, G.W.: Playing Videogames: The Electronic Friend. Journal of Communication 34, 148-156 (1984)

9. Wigand, R.T., Borstelmann, S.E., Boster, F.J.: Electronic Leisure: Video Game Usage and the Communication Climate of Video Arcades. Communication Yearbook 9, 275-293 (1985)

10. Phillips, C.A., Rolls, S., Rouse, A., Griffiths, M.D.: Home Video Game Playing in Schoolchildren: A Study of Incidence and Patterns of Play. Journal of Adolescence 18, 687-691 (1995)

11. Griffiths, M.D.: The Observational Analysis of Adolescent Gaming in UK Amusement Arcades. Journal of Community and Applied Social Psychology 1, 309-320 (1991)

12. Vorderer, P., Hartmann, T., Klimmt, C.: Explaining the Enjoyment of Playing Video Games: The Role of Competition. In: Proceedings of the Second International Conference on Entertainment Computing, pp. 1-9. Carnegie Mellon University, Pittsburgh (2003)

13. Lee, H.C., Wong, S.Y., Wong, Y.Z.: The Massively Multiplayer Online Game in Singapore: Gamers and their Motivations. Final Year Thesis, Nanyang Technological University, Singapore (2006)

14. Burgoon, J.K.: The Unwillingness to Communicate Scale: Development and Validation. Communication Monographs 43, 60-69 (1976)

15. Papacharissi, Z., Rubin, A.M.: Predictors of Internet Use. Journal of Broadcasting and Electronic Media 4, 175-196 (2000)

16. Bruning, S.D.: An Examination of the Social, Psychological, and Communication Variables that Influence User Perceptions of Computer-Mediated Communication Technologies (Doctoral Dissertation, Kent State University) Dissertation Abstracts International (1992)

17. Maslow, A.H.: Towards a Psychology of Well Being. J. Wiley and Sons, New York (1999)

18. Staub, E.: The Psychology of Good and Evil: Why Children, Adults and Group Help and Harm Others. Cambridge University Press, New York (2003)

19. Cheung, P.C., Ma, H.K., Shek, D.T.L.: Conception of success: Their correlates with prosocial orientation and behavior in Chinese adolescents. Journal of Adolescence 21, 31-42 (1998)

20. Eisenberg, N., Miller, P.A., Shell, R., McNalley, S., Shea, C.: Prosocial Development in Adolescence: A Longitudinal Study. Development Psychology 27, 849-857 (1991)

21. Kohlberg, L.: The Philosophy of Moral Development: Moral Stages and the Idea of Justice. Harper \& Row, San Francisco (1981) 
22. Gentile, D.A., Anderson, C.A., Yukawa, S., Ihori, N., Saleem, M., Lim, K.M., Shibuya, A., Liau, A.K., Khoo, A., Bushman, B.J., Huesmann, L.R., Sakamoto, A.: The effects of Prosocial Video Games on Prosocial Behaviors: International Evidence from Correlational, Longitudinal, and Experimental Studies. Personality and Social Psychology Bulletin (in press)

23. Williams, D., Ducheneaut, N., Xiong, L., Yee, N., Nickell, E.: From tree house to barracks: The social life of guilds in World of Warcraft. Games and Culture 1, 338-361 (2006)

24. Dovidio, J.G., Piliavin, J.A., Schroeder, D.A., Penner, L.A.: The Social Psychology of Prosocial Behavior. Lawrence Erlbaum Associates, New Jersey (2006) 